Revista de la red interuniversitaria de estudios sobre las literaturas rioplatenses contemporáneas en Francia

21 | 2020

Glosolalias transplatinas: fantasmas, utopías y ficciones lingüísticas

\title{
Contra el Tratado de Tordesillas. Del neocriollo al portunhol selvagem
}

Contre le Traité de Tordesillas. Du neocriollo au portunhol selvagem

Against the Tordesillas Treaty: From Neocriollo to Portunhol Selvagem

Jorge J. Locane

\section{OpenEdition}

\section{Journals}

Electronic version

URL: http://journals.openedition.org/lirico/9628

DOI: $10.4000 /$ lirico.9628

ISSN: 2262-8339

Publisher

Réseau interuniversitaire d'étude des littératures contemporaines du Río de la Plata

\section{Electronic reference}

Jorge J. Locane, «Contra el Tratado de Tordesillas. Del neocriollo al portunhol selvagem», Cuadernos LIRICO [En línea], 21 | 2020, Publicado el 13 julio 2020, consultado el 29 enero 2021. URL: http:// journals.openedition.org/lirico/9628 ; DOl: https://doi.org/10.4000/lirico.9628

This text was automatically generated on 29 January 2021.

\section{(c) (i) (9)}

Cuadernos LIRICO está distribuido bajo una Licencia Creative Commons Atribución-NoComercialSinDerivar 4.0 Internacional. 


\title{
Contra el Tratado de Tordesillas. Del neocriollo al portunhol selvagem
}

\author{
Contre le Traité de Tordesillas. Du neocriollo au portunhol selvagem \\ Against the Tordesillas Treaty: From Neocriollo to Portunhol Selvagem
}

Jorge J. Locane

\section{Planteo}

1 En "Sopa paraguaia", el prólogo a Mar paraguayo (1992), de Wilson Bueno, Néstor Perlongher sostiene que el libro que introduce constituye un artefacto singular. Según argumenta, se trata de un acontecimiento; más precisamente de un "acontecimento" que "passa pela invenção de uma língua" (7). En las líneas que siguen, no obstante, el poeta neobarroco va a matizar su afirmación al interrogar en qué medida Bueno habría volcado -"recolhido"- en su texto una variante lingüística efectivamente en uso. Sugiere que "Trechos de conversa" habrían ingresado en Mar paraguayo para convertirse en artificio, adquirir soberanía como lengua poética y estatus literario.

De este planteo, se podrían indagar dos puntos. Uno sería la asignación al libro de Bueno de un carácter pionero, tanto en lo que respecta a la "invención" de una lengua a la cuestión de si esa lengua, en efecto, recién se manifiesta como existencia con la publicación de Mar paraguayo- como en lo relativo a si la publicación realmente es adánica, es decir, sin antecedentes o publicaciones previas. Una segunda pregunta, que se corresponde con las reflexiones de Perlongher, buscaría iluminar qué tipo de vínculos se establecen -si es que existe alguno- entre la lengua hablada en la región fronteriza entre Uruguay, Argentina, Brasil y Paraguay, o, vale decir y según se prefiera, la región guaranítica o buena parte de la Cuenca del Río de la Plata, y el acontecimiento textual que representa la concatenación de los monólogos de la marafona del balneário de Guaratuba en Mar paraguayo.

3 En primer lugar, con la perspectiva que dan los más de veinticinco años transcurridos desde su aparición, se podría decir que Mar paraguayo hoy hace sistema con otros textos 
más o menos contemporáneos. El mismo Perlongher (10), de hecho, sugiere un cierto vínculo con Larva. Babel de una noche de San Juan (1983), de Julián Ríos, y con Catatau (1975), de Paulo Leminski. En otro de sus escritos (2000) ${ }^{1}$, dedicado precisamente al uso del portuñol en la literatura, examina un corpus donde ingresan Serafim Ponte Grande (1933), de Oswald de Andrade, Galáxias (1984), de Haroldo de Campos, y Capítulo decapitado (1981), de Héctor Olea. Habría que indagar, si se quiere, en qué medida esta selección configura realmente un corpus, pero, dado que el mismo Perlongher sí da cuenta de una serie de rasgos compartidos, sería excesivo asignarle a Mar paraguayo un carácter adánico en lo que refiere a su emergencia como publicación. En una intervención más reciente, Douglas Diegues establece una genealogía del portuñol que amplía la serie de Perlongher al mismo tiempo que remarca su constante reinvención mediante el uso:

Existem vestígios de lo proto-portunhol selvagem enquanto escritura em algunas páginas de Sousândrade, Oswald de Andrade, Haroldo de Campos, Héctor Olea, Wilson Bueno, Néstor Perlongher, Antonio Fraga... Y posso dizer também que, em ambos lados de la frontera, el pueblo inbenta-lenguas triplefrontero lo sigue inventando enquanto habla. (Daio Borges $2009 \mathrm{Web}$ )

Posiblemente, no obstante, el portuñol en el que se expresa la narradora de Mar paraguayo sí sería una articulación singular, no por su estatus híbrido, pero sí porque ese portuñol -como vamos a ver- no es el mismo que se puede hallar en otros textos o incluso en uso en las diferentes zonas de frontera.

5 A partir de estas ideas iniciales, en las páginas que siguen me propongo indagar qué parentesco se puede establecer entre el neocriollo de Xul Solar, una especie de portuñol literario avant la lettre, y el portunhol selvagem que se viene consolidando desde mediados de los años 80 como forma de expresión estética característica de la zona fronteriza vertebrada por los afluentes del Río de la Plata. Los primeros trazos en neocriollo remontan a la década de 1910, mientras que el portunhol selvagem y, como veremos, sus variantes nominales constituyen un proyecto colectivo que al día de hoy se encuentra en desarrollo. Mar paraguayo y, eventualmente, otros textos como los mencionados por Perlongher representarían instancias bisagra, momentos destacables de (re)articulación. El argumento que hace posible este armado no estaría fundado en una hipótesis del orden de lo gramatical aunque, dado el caso, en efecto se la pueda constatar, sino más bien en una suerte de proclama geopolítica: tanto el proyecto de Xul, a quien se lo suele identificar como un "inventor de lenguajes", como el más reciente se habrían propuesto dar por tierra con el Tratado de Tordesillas para postular una utópica integración latinoamericana por medio de la lengua.

\section{Xul Solar, el neocriollo y su gestación²}

Oscar Agustín Alejandro Schulz Solari nace a fines de 1887 en una Argentina signada por los grandes procesos migratorios que despertarán el recelo de las familias de abolengo patricio. Su padre, de hecho, proviene de Letonia y su madre, de Italia. Nace y crece, pues, bajo el estigma de la migración y el influjo de tres lenguas -el italiano materno, el alemán paterno y el castellano- a las que, si se le da crédito a una nota aparecida en 1956 en la revista Wells. Noticias recogida por Patricia Artundo (91), años más tarde habrán de agregarse el francés, el inglés, el ruso, el portugués y el guaraní y ciertos principios de latín, chino, griego y sánscrito (ver también Schwartz 2005: 35). Transcurrida su infancia, comienza estudios de arquitectura que abandona poco 
después para dar paso a lo que será vocación de por vida: formación autodidacta, constante, desjerarquizada y heterodoxa. Así, va a delinear un perfil "profesional" difícil de clasificar. Jorge Luis Borges, en un retrato hoy muy difundido, lo va a presentar como "Hombre versado en todas las disciplinas, curioso de todos los arcanos, padre de escrituras, de lenguajes, de utopías, de mitologías, huésped de infiernos y de cielos, autor panajedrecista y astrólogo perfecto en la indulgente ironía y en la generosa amistad" (2002: 260). En un episodio de Adán Buenosayres, la novela donde Leopoldo Marechal retrata a su generación, los personajes martinfierristas visitan la parrilla de Ciro. Al ingresar, Adán Buenosayres da a conocer a sus compañeros. Entre ellos se halla "El señor Schultze, astrólogo" (2000: 246). En una entrevista de 1961 publicada en El Mundo, a la pregunta “¿Qué es el señor?”, Xul responde: “Pintor, utopista de profesión" (Artundo 2005: 93).

7 Las notas e investigaciones suelen afirmar que en 1912 se embarca con destino a Europa. Existe, sin embargo, una entrevista que presenta ese episodio crucial en la formación de Xul de otra manera:

Una mañana al salir de su casa, rumbo al empleo, siente de pronto asco por la aplastante rutina que lleva. Gira sobre los talones y echa a andar hacia los diques. Esa misma noche parte de Buenos Aires como marinero clandestino en un carguero cuya meta final es Hong-Kong; parte con una idea que se le va atornillando más y más entre ceja y ceja; convertirse en monje en un monasterio del Extremo Oriente. (Sheerwood 2005: 78)

8 No obstante, por razones algo inciertas -un "malestar repentino", según queda registrado en la entrevista- se ve obligado a abandonar en una escala en Europa el barco que lo trasportaba y a postergar para siempre su plan original de llegar a Extremo Oriente. Londres, así, suele aparecer consignado como su primer lugar de residencia estable (Calero Vaquera 2015: 19; Gasparini 2017: 114) en un periplo que se prolongará hasta 1924 y que, sin duda, inaugurará sensibles mutaciones en nuestro protagonista y sus prácticas. En aquellos años conoce al pintor Emilio Petorutti y al ocultista Aleister Crowley, quienes desde entonces marcan su pensamiento. También de esa época es la transformación de Alejandro Schulz Solari en Xul Solar. Hacia mediados de la década del 10 sus pinturas comienzan a incorporar palabras, mensajes crípticos e inscripciones, como lo testimonia "Sol; Sólo mando" de 1919 con registros como “NUN-/-KA/CES-/-AR". De 1915 es Dos Anjos que, como señala Jorge Schwartz (2005: 35), con el cruce de español y portugués, ya contiene cierto germen de lo que será el futuro neocriollo. También en las cartas dirigidas a sus familiares en Argentina desde su estancia europea se reconoce un paulatino desplazamiento hacia esa lengua en principio artificial que terminará por conceptualizar en una variante, acaso, más acabada recién en su "Conferencia sobre la lengua" pronunciada en Buenos Aires en $1962^{3}$. A modo de ejemplo, Schwartz reproduce una carta dirigida a su padre desde Londres hacia fines de la década: "Mi kerido tata: Esperaba ya este año volverme á la patria desde Londres. Envez estoi aquí desde ha poco i kedaré 2 ó 3 meses. Cansado de tanto salvajismo i atraso ke hai en Europa..." (2005: 36). De acá, entonces, destaco dos puntos: el primero es que el neocriollo -que, aunque ya está en gestación, todavía no termina por definirse como una síntesis entre el español y el portugués- comienza a tomar forma a mediados de los años 10 para seguir evolucionando y ramificándose hasta la muerte de Xul poco después de su conferencia en Buenos Aires. El segundo aspecto remarcable es que su vínculo con Europa está definido por la tensión entre impulsos algo contradictorios: su destino original está en Asia, pero su estancia en 
Europa se prolonga por doce años y nunca retoma su ruta; se queja del salvajismo y el atraso que encuentra, pero tampoco se resuelve a retornar. Finalmente, va a comenzar a concebir una lengua de integración latinoamericana desde Europa y sin pisar en su vida Brasil. Voy a volver sobre esto más adelante.

El proyecto lingüístico de Xul Solar y su evolución están en el centro de varios estudios (Calero Vaquera 2015; Lardone 2017; Lindstrom 1982; Nelson 2012; Rodeiro 2008; Schwartz 2005, entre otros); remito a ellos para mayores precisiones en lo que refiere a la genealogía y a las características formales del neocriollo ${ }^{4}$. Por regla general, estas fuentes subrayan el carácter utópico y humanista que le sirve de sustento: Xul habría concebido su lengua como herramienta de comunicación para estrechar los vínculos y las culturas, en principio, dentro del bloque iberoamericano. En lo que sigue, voy a detenerme solo en algunos aspectos puntuales con la idea de, en un segundo momento, indagar eventuales puntos de contacto entre el neocriollo y la(s) lengua(s) de los proyectos literarios que, más recientemente, ponen en cuestión los órdenes lingüísticos heredados de la expansión colonial europea, mediante una fusión del castellano, el portugués y, con frecuencia, el guaraní.

10 Anoté arriba que, a pesar de su intención, Xul nunca llegó a conocer tierras brasileras (ver Schwartz 2011: 55), de modo que la pregunta por cómo llega a concebir una lengua híbrida entre su castellano nativo y el portugués no es ociosa. Schwartz ha escrito al respecto que el interés de Xul por Brasil se habría comenzado a desarrollar durante su estancia en Europa. Prueba de ello sería que, entre los libros que trajo de vuelta a Argentina en 1924, se encuentra un ejemplar de Brasilien (1920), de Adolf Bieler (Schwartz 2011: 56), o algunas gramáticas de portugués (Ramos Flores 2009: 125). En años posteriores, este interés temprano se habría profundizado y diversificado. Los 58 ejemplares de temas brasileros conservados en su biblioteca revelan, de acuerdo con Schwartz,

a curiosidade insaciável e o leitor contumaz: religiões afro-brasileiras, política e história brasílica, antropologia, geografia, linguística, revistas de época (o Cruzeiro, por exemplo), narrativas de viagem e inúmeros recortes de jornal, com matérias referentes ao Brasil em Amberia. Impressiona a quantidade de livros sobre antropologia, folclore, gramáticas e vocabulários brasileiros e afro-brasileiros. (2011:57)

11 Junto con esta biblioteca, además, habría que considerar otros materiales, como fichas manuscritas donde aparecen consignados vocablos del portugués brasilero. También el subrayado de algunos volúmenes o una carta de 1949 donde manifiesta su interés por participar del Primer Congreso de la Lengua Guaraní-Tupí, en Montevideo, de donde se extrae que no habría limitado sus inquietudes solo al legado europeo. Con la intención de relativizar la autenticidad del interés de Xul por Brasil, sus lenguas y culturas, Maria Bernardete Ramos Flores señala que

Da relação de 58 livros do Núcleo Brasil, na Biblioteca de Xul Solar, 60\% foram editados nas décadas de 1930 e 1940, décadas do conservadorismo brasileiro e do nacionalismo retumbante, da atuação dos intelectuais, mais ou menos, na órbita do governo de Getúlio Vargas, a se dedicarem a diagnosticar a nação, na perspectiva etnográfica e antropológica, com vistas à formação racial do Brasil e à sistematização da língua nacional. (2009: 132)

12 Junto con cuestionamientos de este tipo, tendientes a relativizar el espíritu universalista de Xul, no es raro encontrar abordajes que juzgan su representación del mundo y también sus proyectos lingüísticos, incluido el del neocriollo, de eurocéntricos 
(Nelson 2012: 32; Kefala 2012), ya que sus fuentes primordiales no habrían dejado de ser las que históricamente ofrecían las metrópolis. Si se concede, no obstante, que su viaje a Europa, en sus orígenes, fue ideado para llegar a Asia, y que sus contemporáneos martinfierristas, Borges en primer término, jamás ocultaron su desprecio por las lenguas vernáculas, como el tupí-guaraní, y espurias, como el cocoliche (ver Rodeiro 2008; Gasparini 2017) ${ }^{6}$, lo más atinado es asignarle a Xul un carácter al menos distintivo, realmente singular en lo que refiere a su modo de aproximarse a las alteridades no occidentales. En lo que respecta a su interés por la lengua brasilera, se podría sostener que, en algún momento de sus investigaciones, habría advertido que esa lengua, ese "portugués" trasplantado desde Europa, en realidad estaba permeada por las variantes del guaraní y otras lenguas indígenas. Según informa Schwartz a partir de material de archivo, su fracasada participación en el congreso de Montevideo hubiera versado "sobre palavras indígenas em português e espanhol, e vice-versa" (2011: 60, nota 14), de donde se puede concluir que, para esa época, 1949, Xul ya habría estado consciente de que no solo el portugués, sino también el español americanos, son lenguas de mestizajes transcontinentales; más aún, se podría sostener que quizás incluso habría advertido que el componente guaraní hallable en el español de zonas de la Cuenca del Plata sería un elemento compartido con el portugués del sur de Brasil.

No es motivo de este trabajo indagar en el mayor o menor eurocentrismo de Xul; antes que eso interesa clarificar cómo se gesta y configura el neocriollo. De las observaciones anteriores, entonces, resulta destacable que su estudio del portugués, del guaraní y de los rasgos culturales de Brasil, al no haber tenido contacto directo con el país, se habrían ido desarrollando paulatinamente. Las gramáticas de portugués adquiridas en Europa representan un componente temprano; esas gramáticas, no obstante, constituyeron un modo de acceder a la lengua únicamente en su variante formal, según la evidencia peninsular y no americana ${ }^{7}$. El título de la pintura Dos Anjos estaría compuesto, por lo tanto, de un elemento léxico castellano y de otro portugués, de Portugal. Más tarde, pero aún incluso en Europa, Xul habría comenzado a estudiar las particularidades culturales de Brasil. Ya avanzados los años 20 habría entrado en estrecho contacto con la literatura brasilera, como lo demuestra el ejemplar de Macunaíma (1928) dedicado por Mário de Andrade y subrayado meticulosamente por Xul, en particular en lo que refiere a términos de origen africano y a expresiones coloquiales, que se conserva en su biblioteca (Schwartz 2011: 58). Entre los años 30 y 40, cuando ya había redactado al menos una parte de los San Signos -datan de los años 20 y 30- y publicado sus textos más difundidos en neocriollo, como las traducciones de los Stufen, de Christian Morgenstern ${ }^{8}$, profundiza en el estudio de Brasil, a partir de una ideología que procuraba distinguirlo lingüística y étnicamente de la metrópoli (Ramos Flores 2009: 123-124). De estos estudios concluye que un portugués brasilero depurado de su componente guaraní o sería portugués de Portugal o una lengua artificial desconectada de cualquier uso. En cualquier caso, una que -como pretendía- hubiese resultado inútil para vehicular un proyecto de integración latinoamericana. Independientemente de cuánto espacio puede haberle reservado al guaraní en su diseño del neocriollo -al final habría que considerar que las limitaciones lingüísticas personales son también un factor en juego-, lo cierto es que en lo que va de los primeros trazos en neocriollo a su carta a la organización del congreso en Montevideo hubo una sensible evolución y que, entretanto, Xul no escatimó esfuerzos para familiarizarse con los aportes de origen africano hallables en el portugués de Brasil y con las lenguas indígenas ${ }^{9}$. Esta evolución -que se podría caracterizar como una 
búsqueda permanente- explica también por qué el neocriollo siempre fue una lengua inestable, en constante mutación, incluso mismo dentro de un texto orgánico como lo constituyen los San Signos (ver Nelson 27). Para ejemplificar el carácter inestable, y también porque voy retomarlas en argumentos que voy a presentar más adelante, recupero dos traducciones al neocriollo del poema de Charles Baudelaire "La mort des amants" hechas en diferentes momentos, inciertos, por Xul y reproducidas, a partir de una investigación de Nelson, por Schwartz (2005: 40):

\begin{tabular}{|c|c|c|}
\hline $\begin{array}{l}\text { La mort des amants } \\
\text { Nous aurons des lits pleins d'odeurs légères, } \\
\text { Des divans profonds comme des tombeaux, } \\
\text { Et d'ćtranges flcurs sur des ćtagères, } \\
\text { Ecloses pour nous sous des cieux plus beaux. } \\
\text { Usant à l'envi leurs chaleurs dernières, } \\
\text { Nos deux cœurs scront deux vastes flambeaux, } \\
\text { Qui réfléchiront leurs doubles lumières } \\
\text { Dans nos deux esprits, ces miroirs jumeaux. } \\
\text { Un soir fait de rose et de bleu mystique, } \\
\text { Nous ćchangerons un ćclair unique, } \\
\text { Comme un long sanglot, tout chargé d'adieux; } \\
\text { T:t plus tard un Angc, entr'ouvrant les portes, } \\
\text { Viendra ranimer, fidèle et joyeux, } \\
\text { Les miroirs ternis et les flammes mortes. }\end{array}$ & $\begin{array}{l}\text { La muerte d'os ámantes } \\
\text { Tendremos camas frag'illenas } \\
\text { i divanes tumbihondos } \\
\text { i flores drolas cn cstantes } \\
\text { abrias pa noh, sob ceos mejores } \\
\text { Gastin porfiue xus poscalores } \\
\text { nuestros cuoreh serán dos granteas } \\
\text { quereflejarán xus biluzes } \\
\text { en nuestras mentes, coespejos. } \\
\text { Una tarde rósea i mistia } \\
\text { troncremos un lampo uni, } \\
\text { 'mo largo sollozo } \\
\text { plencarg'io de adioses } \\
\text { I luego un ángel, fiel i jubli } \\
\text { vendrá entreabrin las puertas } \\
\text { pa limpic los cspcjos cmpaninidos } \\
\text { i reanime las flamas muertas. }\end{array}$ & $\begin{array}{l}\text { La muerte dos Keriy } \\
\text { Mui wil ten kâma plen de huêlie leve } \\
\text { Yi diván tumbihondoè } \\
\text { Yi drolflor sur xĉlfo } \\
\text { deselosia pa mui sub keûlo maior } \\
\text { siêl plu' bel } \\
\text { gastin pórfin xus lasti hotie } \\
\text { warmic } \\
\text { naxi cuôr wil sé duo vasti gran tôrche } \\
\text { ke wil reflecte xus bilîx } \\
\text { nen naxi mênte, kwes twin mîrro } \\
\quad \text { hâlma psyûh } \\
\quad \text { soar } \\
\text { Wan soire de rose yi myusti blu } \\
\text { Mwil xanje' un uni' lampo } \\
\text { 'mo diu sobe blitzo } \\
\text { long zasplore } \\
\text { plen cargic de salûto gretic } \\
\text { sauda grûsie } \\
\text { Yi luegó un anjo fiel yi gay } \\
\text { va vene', terabrir lo puêrta } \\
\text { pa limpic li mîrro turbio yi } \\
\text { cleanse a } \\
\text { yi renime li flamo muerta } \\
\text { pa o }\end{array}$ \\
\hline
\end{tabular}

De esta evidencia, ante todo, hay que aceptar que cualquier definición del neocriollo en rigurosos términos gramaticales no resistiría un rápido peritaje textual o, que en el mejor de los casos, solo tendría una validez circunstancial. La definición de Daniel Nelson, quien sin duda mejor ha examinado el neocriollo, es la siguiente: "un lenguaje artificial inventado por el propio autor compuesto básicamente de raíces españolas y portuguesas y destinado a la eventual unificación cultural de Latinoamérica y la creación del nuevo ser humano latinoamericano" (Nelson 2012: 28). El neocriollo, en efecto, es un "lenguaje artificial inventado" en la medida que Xul, al no haber conocido Brasil ni sus zonas de frontera con el mundo de habla hispana, jamás llegó a intuir que existía una comunidad hablante de un portuñol real que le habría permitido sustentar su proyecto en una experiencia concreta. Voy a retomar este punto cuando me refiera al portunhol selvagem. En tanto que la definición de Nelson se encuentra en la introducción a los San Signos, cabría deducir que no contempla la potencial evolución posterior del neocriollo, la que, si se da crédito a la propuesta de Xul de 1949 para la conferencia de Montevideo, posiblemente habría sido más sensible a las lenguas nativas. En cualquier caso, también se podría argumentar que esas "raíces españolas y portuguesas" que conforman la base del neocriollo, al ser de inspiración latinoamericana, tarde o temprano contendrían como supuesto los aportes del guaraní. Al margen de estas consideraciones, creo que la definición de Nelson finalmente acierta al no descuidar el carácter utópico del neocriollo. A falta de mejores recursos, o porque todos presentan limitaciones, parece no haber otra opción que hacer a un lado la dimensión más estrictamente lingüística y centrar la definición en ese trasfondo de voluntarismo geopolítico, de modo que las propias palabras de 1951 de Xul, en 
conversación con Gregory Sheerwood, al respecto, muchas veces citadas, siguen siendo tal vez las que mejor dan cuenta de su lengua:

- ¿Cómo se llama ese novísimo idioma?

-"Criol" o "neocriollo" -prosigue mi enciclopédico entrevistado-. En estos momentos y dentro de sus fronteras, América está dando al mundo convulsionado un gran ejemplo de convivencia, de confraternidad, de mutuo respeto, sobre todo entre los países de origen latino. Qué mejor para consolidar esta tendencia de nuevo concepto de efectiva buena vecindad que un idioma común compuesto por palabras, sílabas, raíces sacadas de las dos lenguas dominantes en Centro y Sudamérica: castellano y portugués, que permitirían, vaya otro ejemplo, convertir la frase "la miró cariñosamente" en la mucho más breve que dice "lakerminu", o "la miró porque quiso" en "la kiermirú". jYo soy decididamente "catrólico"! "Catrólico" es un término "criol", de mi cosecha: designa al individuo simultáneamente católico y astrólogo.

[...]

-Estamos viviendo la época de los grandes bloques: Panamérica, Paneuropa, Panasia -prosigue mi interlocutor-. El "criol" o "neocriollo" podría ser el idioma auxiliar de Panamérica; la "panlingua" sería la lengua complementaria entre los tres bloques. (2005: 76-77)

\section{El portunhol selvagem, sus inflexiones}

Como sugerí en la introducción siguiendo a Perlongher, en 1992 Wilson Bueno "inventa" una lengua que, en algún sentido, quizás ya "había inventado" Xul Solar. Desde luego, nada en esta propuesta debería ser tomado de manera literal, sino más bien como un planteo especulativo destinado a indagar si es posible establecer algún tipo de parentesco entre ambos "acontecimientos". El proyecto del neocriollo queda en suspenso en 1963 con la muerte de Xul. Como vimos con Perlongher y Diegues, es posible identificar un protoportuñol literario cuyos rastros conducen hasta $1933 \mathrm{y}$ Oswald de Andrade. Desde mediados de los años 70 comienzan a aparecer textos en la región sur de Brasil que, acaso inspirados en las vanguardias históricas, exhiben la contaminación lingüística, vale decir, la provocación a la lengua nacional como un valor para las literaturas experimentales. En 1992, con Mar Paraguayo, esas incursiones en territorios de hibridación lingüística cuajan en un texto que se distingue por su particular y sistemático ensamblaje de portugués, español y guaraní. Si el neocriollo de Xul fue un work in progress postulado para superar las limitaciones del español y del portugués, el guaraportuñol de Bueno, desde cierta perspectiva, puede valer por una corrección del proyecto de Xul en la medida en que está fundado en la "intuición" tardía de Xul de que las lenguas europeas trasladadas a América se reconfiguraron como conglomerados lingüísticos con aportes de, al menos, las tradiciones metropolitanas, las vernáculas y las africanas. La "noticia" que introduce Mar Paraguayo es clara al respecto:

Un aviso: el guarani es tan essencial en nesto relato quanto el vuelo del páraro, lo cisco en la ventana, los arrulhos del português ô los derramados nerudas en cascata num solo só suicídio de palabras anchas. Una el error dela outra. Queriendo-me talvez acabe aspirando, en neste zoo de signos, a la urdidura essencial del afecto que se vá en la cola del escorpión. Isto: yo desearia alcançar todo que vibre e tine abaixo, mucho abaixo de la línea del silêncio. No hay idiomas aí. Solo la vertigen de la linguagem. (1992: 13) contar por telefone" (1992: 11). Sería vano intentarlo incluso aunque en efecto una 
línea argumentativa fuese el principio vertebrador del texto. Su razón de ser no está ahí; se encuentra, antes, en el plano formal, en la experiencia verbal que ofrece ese "zoo de signos" donde el guaraní, a diferencia de lo que ocurría en los ejercicios neocriollos de Xul, ahora es "esencial". De hecho, dado que los monólogos de la marafona llevan como título el término "Ñe'ê" (1992: 15) (palabra, lengua, idioma, voz, en guaraní) se podría sostener que Mar Paraguayo es, ante todo, una reflexión práctica sobre la lengua.

Pero ese "zoo de signos" no es únicamente un devaneo estilístico solipsista; pues se proyecta hacia fuera del dominio textual como afrenta. Anota Jens Andermann que "es posible pensar a partir de Mar paraguayo los límites del modelo moderno de construir la nacionalidad desde la literatura, y a ésta como la verdad en la lengua. El entrelenguas de la Triple Frontera que pone en escena el texto de Wilson Bueno desafía de manera performativa a la lengua nacional y a la función que la literatura supo cumplir asociándose a ésta" (2011: 12). Cuestiona, así, los órdenes nacionales, pero también la ideología afirmativa de los purismos lingüísticos y de las lenguas de estirpe metropolitana. El guaraportuñol de Mar Paraguayo -Andermann no vacila en llamarlo "portuñol salvaje"- confunde, cruza, hibridiza las lenguas coloniales hegemónicas $y$, además, las "mancha" con una lengua paria (por lo menos para Brasil, Argentina y Uruguay), vernácula y, además, ajena al prestigioso ascendiente latino. La lengua de Bueno sería, entonces, el neocriollo de Xul atravesado de manera radical -esencial- por esa "lengua menor" que representa el guaraní.

Este sería un punto de encuentro: el guaraportuñol/portuñol salvaje es una corrección superadora del neocriollo. Ambos, en cualquier caso, pretenden dar cuenta de una inscripción geopolítica de frontera, remiten a esa zona donde los Estados nacionales pierden influencia y, con ello, capacidad de interpelar las subjetividades a partir de la pertenencia lingüística. En el caso del segundo, se trata de una fórmula idealista, fundada en el imaginario utópico de Xul, y, por eso, sin arraigo en la experiencia de las comunidades de habla efectivamente existentes; en el primero, por el contrario, estamos frente a una lengua literaria que -como vamos a ver- cada vez más se sentirá reflejo de un lenguaje en uso, como modo de darle voz a subjetividades subalternas, como, de hecho, lo es la marafona, migrante y prostituta.

Es de señalar, también, que en los dos casos se trata de lenguas sin gramática ni ortografía, abiertas al desvío y a la permanente reinvención. Del mismo modo que las variantes en uso del portuñol, al no existir Estados que velen por su corrección, las lenguas de Xul y de Bueno son, en la práctica, ajenas a cualquier fuerza normativa, se desmarcan de la célebre iluminación nietzscheana que le asignaba a la gramática un poder de reglamentación equivalente a Dios. "Não há lei:" -dice Perlongher en referencia al portunhol de Bueno- "há uma gramática, mas é uma gramática sem lei; há uma certa ortografia, mas é uma ortografia errática: chuva e lluvia (grafadas de ambas as maneiras) podem coexistir no mesmo parágrafo, só para mencionar um dos contáveis exemplos" (1992: 9). Mientras que Nelson, por su parte, en lo que refiere al neocriollo, anota que "en los San Signos Xul cambia las reglas fonológicas, ortográficas, morfológicas y sintácticas constantemente, no sólo en la misma visión sino en la misma página, algo que complica inmensamente su desciframiento" (2012: 27).

Pero la aparición de Mar Paraguayo es solo una instancia, sobresaliente, pero una más en la evolución reciente del (guara)portuñol literario. Como adelanté, Andermann lo caracteriza como "portuñol salvaje" y con esto lo inscribe en una corriente que excede por mucho el caso aislado. Desde mediados de los años 80 , diferentes escritores han 
comenzado a identificarse con las variantes del portuñol habladas en la zona de frontera entre Argentina, Brasil, Paraguay y Uruguay -con su centro gravitacional en la Triple frontera- para desarrollar una lengua literaria destinada tanto a problematizar los órdenes nacionales como a prestigiar a las comunidades hablantes de esas lenguas. Aunque de circulación baja y silenciosa, esto es, sin el auspicio de la industria editorial establecida, no se puede calificar ese corpus de inexistente. Tampoco habría que ignorar el carácter programático que, de un tiempo a esta parte, gracias a algunos textos que valen como manifiestos, ha adquirido.

Uno de ellos es, precisamente, el que recupera Andermann en su contribución sobre Mar Paraguayo (2011: 11), una "carta-manifesto" firmada por varios escritores y dirigida a los presidentes Lula y Fernando Lugo en 2008 para que anularan el contrato de Itaipú que regulaba, desde tiempos de dictaduras, la exportación de energía hidroeléctrica de Paraguay a Brasil y acordaran uno nuevo "escrito em portunhol selvagem, la lengua mais hermoza de la triple frontera, pues que nel portunhol selvagem cabem todas las lenguas del Brasil y del Paraguay (incluso las ameríndias) y todas las lenguas del mundo" (Sá Web) ${ }^{10}$.

Otro de ellos es "Porounhol, neolengua de las fronteras en la actual escritura paraguaya", redactado por Cristino Bogado y publicado en 2017. Junto con presentar algunos escritores de la corriente, en este texto Bogado establece una genealogía tanto de la(s) lengua(s) de frontera como de la escritura en portuñol salvaje. También se refiere al término "jopará" que, como el célebre ajiaco de Fernando Ortiz, originalmente definía un plato paraguayo que combina ingredientes heterogéneos para dar lugar a una comida considerada particularmente nutritiva. A continuación señala que

el jopará pasó a definir esa lengua o constructo lingüístico generado a partir del uso que incorpora elementos léxico-gramaticales de español, portugués, guaraní y otras lenguas o dialectos indígenas de la cuenca litoraleña-amazónica, dependiendo su base sintáctica de la zona, en la mayoría de los casos español, pero en algunas regiones (como la frontera Pedro Juan Caballero-Punta Porã) con base portuguesa. En estos últimos casos, el término usado para ser más correctos es poro'unhol o porunhol salvagem, llegando a ser mencionado en un caso analizado como portunhol selbagem. (2017: 196)

Este pasaje informa sobre dos puntos que me interesa destacar: uno, que el portuñol salvaje, una lengua en principio artificial y literaria, se nutre de una experiencia lingüística real, propia de ese extenso territorio fluvial -signado por el fluir; transplatino, diría Perlongher- en el que, a las diversas vertientes vernáculas de la cultura guaraní, se le superponen las que trajeron los múltiples y sucesivos agentes colonizadores: en primer término, los primitivos españoles y portugueses, pero también las colonias menonitas, las de suizos y alemanes, las misiones jesuíticas, y los más recientes de origen árabe o asiático. El segundo punto es que, en su devenir, el portuñol salvaje, que dado el caso se distingue del portuñol a secas porque no se desentiende de esa superposición de elementos culturales y lingüísticos, ha adquirido, de acuerdo con la específica manifestación situada, diversas denominaciones que van del "jopará" al "porunhol salvagem" pasando por diferentes versiones como "portunhol selvagem", "portunhol selbagem", "portuguaraniol salvaje", "portunholito salbahe", "portuñol sauvage" y otros ${ }^{11}$.

Un tercer texto de carácter reivindicativo pertenece a Douglas Diegues. Se trata de un poema titulado "Triple frontera selvagem" (2014) cuyos primeros versos, en un gesto 
metalingüístico, anclan la lengua del poema, el portunhol selvagem, al espacio de tránsito concreto que representa la Triple frontera:

Bienvenidos a la Triple frontera selvagem, amables lectores,

kontradicciones frias y dicciones kalientes,

koreanos, árabes, chinos, paraguayos, brasilenhos,

vos saludam en uma lengua que non existe como idioma

pero que puede ser escrita y hablada

como el amor

que voce mai ou menos puede entender

mismo que non entenda puerra ninguma. (211)

Este mismo poema aparece comentado por Pablo Gasparini en su texto ya citado dedicado, en principio, a la "liminal transnacionalidad de Xul". Su observación, sin profundizar en las eventuales relaciones entre el neocriollo y el portunhol selvagem, es que

La creciente emergencia de una poética con visos fronterizos en Brasil, Argentina y Paraguay ${ }^{12}$ puede constituir, entre otros materiales, un buen ejemplo de la imaginaria superposición de espacios aparentemente discontinuos, espacios que lingüística e identitariamente resignan sus gramáticas y órdenes en pro de un tránsito sin jerarquías. En 'Triple frontera selvagem', el poeta brasileño Douglas Diegues, hace del 'Puente de la amistad' un espacio que vale menos como pasaje (al estilo cortazariano), que como un transitar que es, en sí mismo, un territorio. (2017: 124)

A esta poética de tránsito, que alienta las desjerarquizaciones y que se pretende atenta a los usos concretos, contribuyen, junto con la escritura de Bueno, de Diegues y Bogado, de diferentes maneras, Maggie Torres, Marcelo Silva, Jorge Kanese, Xico Sá, Ronaldo Bressane, Edgar Pou, Miguelangel Meza, Joca Reiners Terron, Fabián Severo y otros.

Nacido en Asunción en 1947, Jorge Canese, Jorge Kanese o Xorxe Kanexe, según se prefiera, viene cultivando su variante del portunhol selvagem desde mediados de los años 80 con libros como Kantos del akantilado (1987) de modo que hoy vale como una referencia ineludible de la poética. En una entrevista de 2010 con Timo Berger, se identifica como cultor de una "pornovanguardia" que retoma a Dadá, y dice estar agradecido de haber nacido en Paraguay porque, de lo contrario, no hubiera podido "Escribir lo que escribo. [...] Inventar un lenguaje", con lo que, a su manera, se estaría inscribiendo en la familia de los "inventores" Xul y Bueno. Entre los poemas en prosa que componen su "Los halcones rosados" (2010) como parte de la trilogía La conspiración de los ginecólogos (2006) se encuentra el siguiente:

Bombachita kunu'ü. Agacháte nena linda, agacháte che mamá, a ver si poro-aldescuido ta-ma 'ëmi nde tatú. Takoari ñamanone, tamanomi che-kambá. Bombachita-a-motare amanoseté, ahechane hakure nde cuarto-ipyguiolado. Por siempre-ngo a consentí (vyroreí de las vyrezas), ahëtusé de las cortezas, ykere nde tatu' i. Maiteí cheve: peteí, la cabecita amoïro, el restotembo-reí. Bombachita kunu u, koanga añepyrü el baile con insistencia: anichéne la pendencia... che intrumento nderaihú. Las chifladuras tienen sus cosas, su mate amargo, su tereré lavado. ¿Erótica häu? Juro que me alzaré en enero. La concha del jabalí rompió el condón rutilante. Pobre tipo chera'́a comentó Engelberta, la que siempre anduvo (obviamente) con la concha abierta. (2006:58)

Como se observa, esta variante de portunhol selvagem, a diferencia de la de Diegues, por ejemplo, se nutre, principalmente, del castellano y del guaraní para, de ahí, derivar neologismos. Se trata, por lo tanto, de una fórmula que remite al habla del interior de Paraguay y no tanto a la zona de frontera con Brasil. Con esto lo que quiero sugerir es 
que, en última instancia, al no existir recursos para su estandarización, habría tantos portunholes selvagems como usuarios. Todos pueden ser subsumidos bajo una misma categoría en la medida que se presentan como híbridos lingüísticos, vinculados, en principio, a la zona fronteriza entre Brasil y algunos de sus países colindantes, y que, a diferencia del portuñol, no disimulan el aporte guaranítico en tanto componente nativo.

Un último ejemplo permite visualizar mejor este carácter aleatorio del portunhol selvagem. Joca Reiners Terron publicó en 2008 en Yiyi Jambo, la editorial cartonera especializada en literatura "transfronteriza"13 más bien transcreada- al portunhol selvagem bajo el título Transportuñol borracho. El subtítulo aclara que se trata de 15 joyitas bêbadas de la poesía universal contrabandeadas al portuñol salbaje por Joca Reiners Terron. Los poemas son de Ikkyū Sōjun, Hans Magnus Enzensberger, Jim Dodge, Malcolm Lowry, Stephen Dobyns, Richard Brautigan y Raymond Carver. De este último, una de las traducciones es la siguiente (24):

\begin{tabular}{|c|c|}
\hline The Best Time Of The Day & La mejor hora del día \\
\hline Cool summer nights. & Noches frescas di verano. \\
\hline Windows open. & Ventanas abiertas. \\
\hline Lamps burning. & Lámparas accsas. \\
\hline Fruit in the bowl. & Frutas en la cesta. \\
\hline And your head on my shoulder. & Y su cabeza en mío ombro. \\
\hline These the happiest moments in the day. & Los momento más felices del día. \\
\hline $\begin{array}{l}\text { Next to the early morning hours, } \\
\text { of course. And the time } \\
\text { just before lunch. } \\
\text { And the afternoon, and } \\
\text { carly cvening hours. } \\
\text { But I do love }\end{array}$ & $\begin{array}{l}\text { Cercano a las primeras horas de la mañana, } \\
\text { claro. Y los instantes } \\
\text { antes del almoço. } \\
\text { Y la tarde, y } \\
\text { el anochecer. } \\
\text { Ah, como amo }\end{array}$ \\
\hline $\begin{array}{l}\text { these summer nights. } \\
\text { Even more, I think, } \\
\text { than those other times. } \\
\text { The work finished for the day. } \\
\text { And no one who can reach us now. } \\
\text { Or ever. }\end{array}$ & $\begin{array}{l}\text { Las noches de verano. } \\
\text { Aún más, pienso yo, } \\
\text { ki las de otras estações. } \\
\text { Terminadas las tarefas del dia. } \\
\text { Y niguém que pueda nos alcançar ahora. } \\
\text { Ou jamás. }\end{array}$ \\
\hline
\end{tabular}

En esta variante -como se advierte- el guaraní pierde importancia y, así, el portunhol selvagem pareciera volver a resolverse como portuñol. Pero la selección de Terron, además de servir como contraste, también interesa por otra razón. Al igual que la operación de Xul con Baudelaire ya presentada arriba, lo que ofrece esta propuesta es una desjerarquización de la tradición poética, una apropiación tendiente a incorporar la poesía con reconocimiento internacional al corpus de la escrita en esa lengua espuria, desafiante de los Estados y las lenguas coloniales, que es el portunhol selvagem.

Para cerrar, a partir del paralelismo entre las traducciones de Xul y las de Terron, me interesa destacar la heterogeneidad formal de sus lenguas. Llama la atención que, si bien tanto el neocriollo como el portunhol selvagem se autoproclaman como híbridos de español y portugués, con mayor o menor aporte de guaraní, los resultados de las traducciones sean, no obstante, tan diferentes. Las lenguas a las que están traducidos Morgenstern, Baudelaire y, por otro lado, Carver y los demás poetas de la publicación de Terron son, ciertamente, por completo disímiles. Pero también lo son, y de manera 
notable, las de las dos traducciones del soneto de Baudelaire hechas por Xul al neocriollo. Más aún, lo que más sorprende -como ya quedó señalado- es que el neocriollo ni siquiera es consistente en una página de los San Signos y que el portunhol selvagem ni siquiera lo es en una de Mar Paraguayo. De acá, pues, lo que cabe concluir es que, si hay algo que, definitivamente, emparenta al neocriollo con el portunhol selvagem es su radical mutabilidad.

\title{
Conclusiones
}

El itinerario que va de 1915, cuando una escritura en una lengua que fusionaba el castellano y el portugués irrumpe en las pinturas de Xul Solar, hasta las manifestaciones más recientes del portunhol selvagem o alguna de sus variantes describe, fundamentalmente, la evolución de una afrenta. Aun cuando sus efectivos valores de uso resulten difíciles de dilucidar, las lenguas de ensamblaje que son el neocriollo y la de Wilson Bueno y Douglas Diegues toman partido por un lugar de entrecruzamientos donde las prescripciones estatales pierden poder de interpelación. Anota Adrián Cangi que

\begin{abstract}
Socavar las miserias de los estados nacionales supone doblegar la voz monocorde y homogénea de una forma madura, en favor de las hablas migrantes, de los balbuceos híbridos y confusos de las mezclas, de las agrupaciones inverosímiles del cocoliche o de las lenguas en tránsito territorial. Las lenguas en tránsito son hybris acéfalas, que instalan la parodia entre zonas de vecindad lingüística, sin identificación simbólica, sin mímesis formal, sólo recuperando el fluido sonoro como base constructiva en la vecindad. (2000: 266)
\end{abstract}

Este, el de reivindicar las contaminaciones y perturbar el purismo monolingüe del Estado, sería un primer principio articulador que permitiría ubicar al neocriollo y al portunhol selvagem juntos en una línea argumentativa continua.

Si de informar acerca de sus diferencias se trata, habría que decir que, mientras que el neocriollo fue una lengua de un usuario único, en el caso del portunhol selvagem estamos frente a un proyecto de factura colectiva. Es cierto que algunos de los escritores mencionados en este trabajo quizás refutarían su pertenencia a una poética compartida con Douglas Diegues, o que los rasgos formales de las escrituras de Jorge Kanese y Fabián Severo, por ejemplo, pueden resultar sensiblemente disímiles, pero también es innegable que muchos de ellos son parte de una misma estructura afectiva y de activismo cultural, y que no es raro ya sea que ellos mismos visibilicen la pertenencia o que se los asocie. Como se vio y con todas las diferencias que se puedan encontrar en los atributos de sus lenguajes literarios, mientras que Diegues vale por el principal cultor del "portunhol selvagem", Andermann caracteriza el de Bueno como "portuñol salvaje", y lo que hace Terron en su selección de poesía "universal" es traducirla al "portuñol salbaje". De modo que, si la lengua de Xul fue, ante todo, un proyecto individual que como herramienta de comunicación apenas pudo ser utilizada para escribir algunas cartas, hacer un puñado de traducciones experimentales y redactar unas visiones que recién vieron la luz pública una vez que fueron traducidas por Nelson al castellano, en el caso de la lengua fronteriza en la que vienen escribiendo algunos escritores del sur de Brasil, de Paraguay y del norte de Argentina y Uruguay desde los años 80 a la actualidad, con un texto como Pindó Kuñakaraí (2018), de Cristian Bogado, se trata de un proyecto colectivo, todavía en proceso y, sin duda, más orgánico 
que el de Xul; incluso con plataformas editoriales como Yiyi Jambo, Jakembo Editores y La Editora de Los Bugres.

Esta posibilidad de articular un proyecto compartido entre escritores con diferentes trasfondos nacionales y a largo plazo se sostiene en la toma de conciencia y reivindicación de que la zona de frontera también puede ser un lugar de enunciación y de que en ese territorio donde los órdenes nacionales comienzan a vacilar, de hecho, existen comunidades hablantes que hacen del portuñol, con más o menos guaraní, con más o menos español, con más o menos portugués, una lengua en uso. A la luz de esta evidencia, habría que anotar que lo que hizo Xul fue concebir una lengua de laboratorio, fundada en una permanente especulación gramatical y, por lo tanto, ajena a esas comunidades de habla que le podrían haber otorgado sustentabilidad a su proyecto. Debido a que su experiencia biográfica está marcada por el vacío de que, a pesar de su interés, jamás entró en contacto directo con Brasil en general y con la zona de frontera en particular, solo mediante la investigación, su lengua fue evolucionando para alejarse de Europa y aproximarse a los usos americanos. Finalmente, también llegó a intuir que el guaraní -como sinécdoque del aporte vernáculo- de ninguna manera podría haber sido un componente prescindible de su utopía lingüística.

He intentado destacar que no es posible identificar principios gramaticales $u$ ortográficos que operen como mecanismos reguladores del neocriollo y del portunhol selvagem, ya sea si se quiere imaginar un continuo que las conecte o si se toma un elemento aislado, una evidencia textual en alguna de las dos lenguas, como objeto de análisis. Cualquiera que quiera convertirse en su usuario, aunque sea circunstancial, puede apropiárselas y recrearlas de acuerdo con su propia predisposición a la inventiva (a)gramatical. Se trata, por lo tanto, de dos lenguas desreguladas y en permanente mutación. Consideradas desde este punto de vista, sería inconducente tratar de establecer paralelismos entre una y otra. Si como señalan Perlongher y Nelson, respectivamente, ambas se caracterizan por la inestabilidad; si mismo en una página en neocriollo o en una en portunhol selvagem no hay norma, sería en absoluto vano intentar encontrar recurrencias formales que conduzcan de una a la otra. Y, sin embargo, se podría decir que ambas pertenecen a una familia; a una organizada desde luego no a partir de la gramática sino de la proclama geopolítica que supone una experiencia compartida y un destino común para los sujetos latinoamericanos. Su rasgo común más trascedente sería, pues, el carácter utópico y que, en tanto inflexiones abiertamente transligüísticas, cuestionan el Tratado de Tordesillas y, con ello, las determinaciones geopolíticas de cuño colonial. El rediseño de la geografía americana por parte de los poderes coloniales implicó una dislocación de los universos culturales precolombinos. La región guaranítica en particular presentaba una fisionomía cultural y lingüística relativamente continua que fue quebrada por la violencia colonial. El neocriollo y el portunhol selvagem, cada uno a su modo, intervienen en el dominio simbólico para contaminar los órdenes culturales que las metrópolis, a partir de intereses económicos envestidos de arengas puristas, dispusieron mantener separados: fusionan el español y el portugués y, al mismo tiempo, tienden a inocular en ese híbrido de por sí sospechoso la mácula de la lengua indígena inferiorizada y sometida. 


\section{BIBLIOGRAPHY}

Andermann, Jens, “Abismos del tercer espacio: Mar paraguayo, portuñol salvaje y el fin de la utopía letrada”, Revista Hispánica Moderna, nº 64, 1, 2011, p. 11-22.

Artundo, Patricia (ed.), Alejandro Xul Solar. Entrevistas, artículos y textos inéditos, Buenos Aires, Corregidor, 2005.

Berger, Timo, “¿Qué dice un lenguaje que no se puede entender? Una entrevista con el autor paraguayo Jorge Kanese”, Humboldt, 2010. Web. Consultado el 20 de diciembre de 2019.

Bogado, Cristino, "Porounhol, la neolengua de las fronteras en la actual escritura paraguaya", Luvina no 87-88, 2017, p. 194-208.

Borges, Jorge Luis, “A Xul Solar”, Textos recobrados 1931-1955, Buenos Aires, Emecé, 2002 [1949], p. 260.

Bueno, Wilson, Mar paraguayo, São Paulo, Iluminuras, 1992.

Calero Vaquera, María Luisa, “Las lenguas inventadas de Xul Solar (1887-1963): ‘Una bella locura en la zona del lenguaje”, Revista argentina de historiografía lingüística, nº VII, 1, 2015, p. 17-30.

Cangi, Adrián, “Una poética bastarda”, Tsé Tsé, nº 7-8, 2000, p. 265-273.

Daio Borges, Julio, “Douglas Diegues”, Digestivo cultural, 1 de enero de 2009. Web. Consultado el 20 de diciembre de 2019.

Degli Atti, Francesca, "Considerações acerca do movimento do Portunhol selvagem: o paradigma da osmose e a resistência cultural”, Babilónia: Revista Lusófona de Línguas, Culturas e Tradução, ํㅡ13, 2013: p. 47-72.

Diegues, Douglas, “Triple frontera selvagem”, Diana Araujo Pereira (ed.), Cartografia imaginaria da tríplice fronteira, São Paulo, Dobra universitário, 2014, p. 211-216.

Freitas Bittencourt, Rita Lenira de, "Editoras cartoneras e espaços de fronteira: encontros e desencontros", Anais do XV Congresso Internacional da Associação Brasileira de Literatura Comparada, 2017. Web. Consultado el 20 de diciembre de 2019.

Gasparini, Pablo, "Sobre la liminal transnacionalidad de Xul (o del desborde lingüísticovanguardista de una territorialidad latinoamericana)", Boletín Hispánico Helvético nº 29, 2017, p. 105-128.

Kanese, Jorge, La conspiración de los ginecólogos, Asuncion, Jakembo, 2016.

Kefala, Eleni, “'La gran América Ibérica': humanismo y utopía en los años veinte. El caso de Xul Solar", Revista canadiense de estudios hispánicos, nº 36, 3, 2012, p. 457-479.

Lardone, Mariana Inés, "La lengua Xul Solar: la escritura neocriolla como acto de evocación”, Orbis Tertius, nº 22, 25, 2017. Web. Consultado el 20 de diciembre de 2019.

Lindstrom, Naomí, "El utopismo lingüístico en Poema de Xul Solar”, Texto Crítico, no 24-25, 1982, p. 242-255.

Locane, Jorge J., "Disquisiciones en torno al portunhol selvagem. Del horror de los profesores a una 'lengua pura", Perífrasis. Revista de literatura, teoría y crítica, no 6, 12, 2015, p. 36-48.

---, “A propósito de unas traducciones ludiserias de Xul Solar”, alba. lateinamerika lesen, no 5, 2014, p. 28-35. 
Marechal, Leopoldo, Adán Buenosayres, Buenos Aires, Agea, 2000 [1948].

Nelson, Daniel E., "Un texto proteico: los San Signos de Xul Solar", Xul Solar, Los San Signos. Xul Solar y el I Ching, Buenos Aires, El hilo de Ariadna, 2012, p. 21-102.

Pagni, Andrea, "Xul Solar, un traductor en dos escenas de traducción”, Mónica Marinone y Gabriela Tineo (ed.), Latinoamérica entre lenguajes y lenguas, Mar del Plata, Edudem, 2018, p. 45-75.

Patiño Wandurraga, Nayda Katherine, Mar Paraguayo y Viralata: exaltación poética del portuñol, una lengua con dinámica propia, Foz do Iguaçu, Unila, 2018. Web. Consultado el 20 de diciembre de 2019.

Peres Alós, Anselmo, “Portuñol selvagem: da 'língua de contato’ à poética da fronteira”, Cadernos de Letras da UFF, ํㅡㄴ 45, 2012, p. 283-304.

Perlongher, Néstor, “El portuñol en poesía”, Tsé Tsé, nº 7-8, 2000, p. 254-259.

---, “Sopa paraguaia”, Wilson Bueno, Mar paraguayo, São Paulo: Iluminuras, 1992, p. 7-11.

Ramos Flores, Maria Bernardete, "Xul Solar e o Brasil. Sobre uma biblioteca muito particular", Eadem Utraque Europa, no 9, 2009, p. 119-154.

Rodeiro, Matías: “Xul. Más allá del idioma (de los argentinos)”, Horacio Gónzalez (org.), Beligerancia de los idiomas. Un siglo y medio de discusión sobre la lengua latinoamericana, Buenos Aires: Colihue, 2008, p. 185-252.

Sá, Fátima, "Karta-manifesto-del-amor-amor-em-portunhol-selvagem", o Globo, 17 de agosto de 2008. Web. Consultado el 20 de diciembre de 2019.

Schwartz, Jorge, “Xul/Brasil: imaginários em diálogo, Revista do Instituto de Estudos Brasileiros, no 53, 2011, p. 53-67.

---, "Sílabas las estrellas compongan: Xul y el neocriollo", Xul Solar. Visiones y revelaciones, Buenos Aires/São Paulo: MALBA-Fundación Constantini/Pinacoteca do Estado de São Paulo, 2005, p. $35-47$.

Sheerwood, Gregory, “Gente de mi ciudad: Xul Solar, campeón mundial de panajedrez y el inquieto creador de la 'panlingua"', Patricia Artundo (ed.), Alejandro Xul Solar. Entrevistas, artículos y textos inéditos, Buenos Aires: Corregidor, 2005 [1951], p. 75-80.

Solar, Xul, Los San Signos: Xul Solar y el I Ching, Buenos Aires: Fundación Eduardo F. Constantini/ Fundación Pan Klub, 2012.

---, “Conferencia sobre la lengua”, Patricia Artundo (org.), Alejandro Xul Solar. Entrevistas, artículos y textos inéditos, Buenos Aires, Corregidor, 2005 [1962], p. 198-204.

Terron, Joca Reiners, Transportuñol borracho. 15 joyitas bebadas de la poesía universal contrabandeadas al portuñol salvaje, Asunción, Yiyi Jambo, 2008.

\section{NOTES}

1. "El portuñol en la poesía", originalmente, fue presentado como ponencia en el Encuentro de Profesores de Español del Estado de São Paulo en 1984.

2. Este apartado retoma, corrige y amplía mi artículo "A propósito de unas traducciones ludiserias de Xul Solar" (2014).

3. En esta conferencia, Xul propone, en realidad, una lengua de alcance panamericano (198), capaz de maridar el español, el portugués y el inglés, como las lenguas más extendidas en América. 
4. La publicación en 2012 de los San Signos, el texto más orgánico redactado en neocriollo, posiblemente obligaría a replantear algunos de los postulados formulados con anterioridad. Un análisis de este tipo sería, no obstante, motivo de otro trabajo.

5. En este artículo Ramos Flores discute los planteos de Schwartz acerca de la relación de Xul con Brasil. De este último, existe una versión de 2005. La que yo cito es posterior a la publicación de Ramos Flores.

6. De acuerdo con Daniel Nelson, "su actitud inclusiva hacia los indios americanos y los mestizos difiere radicalmente de la actitud despectiva hacia ellos que se aparenta en el anónimo "Manifiesto de "Martín Fierro" comúnmente atribuido a Oliverio Girondo" (2012: 35).

7. "Entre os 58 livros situados no Nucleo Brasil encontra-se algumas gramaticas adquiridas na sua fase europeia, tais como, Gammatichetta Portuguese, uma gramatica publicada em Milano, para um italiano aprender portugues. Da mesma forma, Pequeno livro de lectura portugueza, livro de poesias e contos portugueses com explicacoes gramaticais e tradução de termos e expressoes para o italiano" (Ramos Flores 2009: 125).

8. Al respecto, véase Pagni 2018.

9. De hecho, en una entrevista/retrato publicada en 1961 se consigna que Xul "Ha inventado [...] un idioma, el 'neocriollo'. Mezcla de español, portugués, guaraní, enriquecido últimamente con el inglés" (2005: 93).

10. Al respecto, véase también Degli Atti 2013; Locane 2015; Peres Alós 2012.

11. Para simplificar, me voy a quedar con la fórmula portunhol selvagem para englobar todas estas variedades, siempre bajo la premisa de que incluyen, en diferentes medidas, español, portugués y guaraní.

12. Como ya sugerí, lo adecuado sería incluir en esta cartografía también la zona de frontera de Uruguay porque, aunque con sus especificidades, también allí se ha gestado un cierto guaraportuñol literario. Véase Locane 2015 y Patiño Wandurraga 2018.

13. Al respecto, véase Freitas Bittencourt 2017.

\section{ABSTRACTS}

This article carries out a parallel analysis of neocriollo, the imaginary language conceived by Xul Solar, and portunhol selvagem, a literary language whose epicenter is located at the Triple Frontier (between Argentina, Brazil and Paraguay) and which, since the mid-1980s, has been used in the southern border of Brazil. We conclude that, although both languages are characterized by their instability and by the lack of a grammar that could settle them -they don't share formal features-, they can still be located within the same genealogy, insofar as, by merging the imperial languages and infecting them with Guarani, they disturb the colonial order established by the Treaty of Tordesillas, functioning thus as a utopian geopolitical proclamation.

El presente trabajo propone un análisis en paralelo del neocriollo, la lengua imaginaria concebida por Xul Solar, y el portunhol selvagem, una lengua literaria que, con epicentro en la Triple Frontera, se viene cultivando en la zona de la frontera sur de Brasil desde mediados de los años 80. Se concluye que, si bien ambas se caracterizan por la inestabilidad y la falta de una gramática que las fije y que, por lo tanto, no poseen rasgos formales comunes, sí pueden ser ubicadas en una misma genealogía en la medida que, al fusionar las lenguas imperiales y contaminarlas con 
guaraní, perturban el orden colonial establecido con el Tratado de Tordesillas y operan, así, como una utópica proclama geopolítica.

Cet article propose une analyse en parallèle du neocriollo, la langue imaginaire conçue par Xul Solar, et du portunhol selvagem, une langue littéraire dont l'épicentre se trouve à la Triple Frontière (entre l'Argentine, le Brésil et le Paraguay) et qui, depuis le milieu des années 1980, est pratiquée dans la frontière sud du Brésil. Nous concluons que, bien que les deux soient caractérisées par leur instabilité et par le manque d'une grammaire capable de les fixer - elles n'ont pas de traits formelles en commun -, elles peuvent tout de même être situées à l'intérieur d'une même généalogie, dans la mesure où, en fusionnant les langues impériales et en les contagionnant de guarani, elles perturbent l'ordre colonial établi par le Traité de Tordesillas et fonctionnent, de cette manière, comme une utopique proclamation géopolitique.

\section{INDEX}

Palabras claves: neocriollo, portunhol selvagem, frontera, geopolítica, lengua

Keywords: neocriollo, portunhol selvagem, border, geopolitics, language

Mots-clés: neocriollo, portunhol selvagem, frontière, géopolitique, langue

\section{AUTHOR}

JORGE J. LOCANE

Universidad de Oslo

jjlocane@gmail.com 